can help in the proper evaluation of the standard errors for all parameters used. Whereas in computing the radial distribution curve all the experimental intensities are included with equal weights, even though some of them are less reliable because of certain reasons for which corrections often cannot easily be made, the intensities can be given appropriate weights in the least-squares treatment so that all the experimental data are amalgamated to yield a set of molecular data with specified errors.

\section{ACKNOWLEDGMENT}

The authors gratefully acknowledge many helpful discussions with Dr. M. Kimura.

\title{
Approximate Isotopic Frequency Rule and Its Application to the Spectra of Complex Molecules
}

\author{
S. KRIMM \\ Harrison M. Randall Laboratory of Physics, The University of Michigan, Ann Arbor, Michigan
}

(Received January 4, 1960)

\begin{abstract}
An isotopic frequency rule is derived which is valid to a good approximation for individual modes of fully substituted hydrogen-containing groups in a complex molecule. It is given by $\lambda^{i} / \lambda=1-\left(\Sigma_{j} \Delta T_{j} / \rho T\right)$, where $\lambda=4 \pi^{2} \nu^{2}, \rho=m^{i} / m=$ ratio of the isotopic to the normal mass, $T=$ total kinetic energy associated with the vibration, and $\Sigma_{j} \Delta T_{j}=$ differential kinetic energy associated with the substituted atoms. When applied to $\mathrm{H}_{2} \mathrm{O}$ and $\mathrm{NH}_{3}$ this rule reproduces frequency ratios to better than $0.5 \%$. The rule is used to predict the frequency ratios for the various modes of the $\mathrm{CH}_{2}$ group, and with illustrations from the infrared spectra of high polymers it is shown how this rule can be of assistance in making assignments and in providing information on the separability of group vibrations.
\end{abstract}

\section{INTRODUCTION}

$\mathbf{T}$ HE detailed analysis of the infrared and Raman spectra of a complex molecule (such as, for example, a high polymer) can provide significant information about the structure of the molecule and about the nature of its intramolecular and intermolecular forces. Such an analysis requires that a complete assignment be made of the observed bands in the spectrum to the predicted modes of vibration of the molecule. One of the techniques for identifying the origin of spectral bands, and thereby assisting in the assigning of these bands, is isotopic substitution. For example, when deuterium is substituted for hydrogen in a molecule, we can expect that the frequencies of those modes involving the motions of hydrogen atoms will be lowered. Thus, qualitatively, the shift of a band to lower frequencies upon such isotopic substitution serves to identify the band as originating to some extent in a hydrogen mode. In this article we are interested in investigating what can be said in greater detail about the normal mode from the quantitative magnitude of the frequency shift ratio.

From the theory of small vibrations it is possible to deduce an exact rule relating the frequencies of the normal and the substituted molecules, viz., the TellerRedlich product rule. ${ }^{1,2}$ The difficulty with using this

${ }^{1}$ G. Herzberg, Infrared and Raman Spectra (D. Van Nostrand Company, Inc., Princeton, New Jersey, 1945), p. 231.

2 E. B. Wilson, Jr., J. C. Decius, and P. C. Cross, Molecular Vibrations (McGraw-Hill Book Company, Inc., New York, 1955), p. 183. rule in the analysis of complex molecules is that it relates the products of all frequencies belonging to the same symmetry species. Therefore, if a large molecule has low symmetry, as is usually the case, we only obtain a relationship between a large number of frequencies. Ideally, we would like a relation between only two frequencies, that of a given mode in the normal molecule and the frequency of the corresponding mode in the substituted molecule. Although such relations have been derived for small mass changes, ${ }^{3}$ they are not very satisfactory when the mass alters by a factor of two, as when deuterium is substituted for hydrogen. Since the latter substitution is the most useful one in the study of complex molecules, we are concerned here with the derivation of a rule which, even if approximate, will be valid for such cases. Specifically, we wish to determine, for example, the frequency shift ratio for each of the modes of a $\mathrm{CH}_{2}$ group when both hydrogen atoms are replaced by deuterium atoms.

\section{THEORY}

For a differential mass change in a molecule, the theory of small vibrations provides an exact expression for the differential frequency shift. It can be shown ${ }^{4,5}$ that this is given by

$$
d \lambda_{k} / \lambda_{k}=-\sum_{j} d T_{j} / T_{k},
$$

\footnotetext{
${ }^{3}$ Work cited in footnote 2, p. 188.

${ }^{4}$ E. Teller, Hand- und Jahrb. Chem. Physik 9(II), 43 (1934).

${ }^{5}$ H. J. Bernstein, Can. J. Chem. 29, 284 (1951).
} 
where

$$
\lambda=4 \pi^{2} v^{2} .
$$

In these equations, $\nu$ is the frequency, $\lambda_{k}$ is the value of $\lambda$ associated with the $k$ th mode of the normal molecule, $T_{k}=\sum m_{i} \dot{q}_{k l}{ }^{2}=$ total kinetic energy associated with the $k$ th vibration in the normal molecule, and $\sum_{j} d T_{j}=\sum_{j} d m_{j} \dot{q}_{k j}{ }^{2}=$ the differential kinetic energy in the $k$ th mode associated with the substituted atoms $j$ when a mass change $d m_{j}$ is made. If we assume the substitution of all atoms of one kind in the group, viz., hydrogen in the present case, then Eq. (1) provides an exact expression for the derivative of $\lambda_{k}$ with respect to $m$, the mass of the hydrogen atom:

$$
d \lambda_{k} / d m=-\lambda_{k}\left(\sum_{j} \dot{q}_{k j}{ }^{2} / T_{k}\right)
$$

If these exists an expression for $\lambda_{k}$ as a function of $m$, then $\mathrm{Eq}$. (3) can be used to obtain the finite change in $\lambda, \Delta \lambda_{k}$, associated with a finite change in mass, $\Delta m$.

No exact expression exists for $\lambda_{k}(m)$ which is generally true for all molecules. However, for the kinds of small molecules or groups which concern us (e.g., $\mathrm{OH}, \mathrm{CH}_{2}, \mathrm{CH}_{3}$ ), it can be shown (see Appendix) that to a good approximation

$$
\lambda_{k}=a_{1}+\left(a_{2} / m\right),
$$

where $a_{1}$ and $a_{2}$ are constants. If we designate by a superscript $i$ quantities relating to the isotopically substituted molecule, then Eq. (4) leads to

$$
\Delta \lambda_{k} / \Delta m \equiv\left(\lambda_{k}{ }^{i}-\lambda_{k}\right) /\left(m^{i}-m\right)=-\left(a_{2} / m m^{i}\right) .
$$

Since it also follows from Eq. (4) that

$$
d \lambda_{k} / d m=-\left(a_{2} / m^{2}\right)
$$

Eq. (5) can be written

$$
\Delta \lambda_{k} / \Delta m=(\rho)^{-1}\left(d \lambda_{k} / d m\right),
$$

where

$$
\rho=m^{i} / m \text {. }
$$

By substituting Eq. (3) into Eq. (7), we find that

$$
\left(\lambda_{k}{ }^{i}-\lambda_{k}\right) / \Delta m=-\left(\lambda_{k} / \rho\right) \cdot\left(\sum_{j} \dot{q}_{k j}{ }^{2} / T_{k}\right) \text {. }
$$

From this it follows that

$$
\lambda_{k}{ }^{i} / \lambda_{k}=1-\left(\sum_{j} \Delta T_{j} / \rho T_{k}\right)
$$

where $\sum_{j} \Delta T_{j}=\sum_{j} \Delta m \dot{q}_{k j}{ }^{2}$. Equation (10) is the basic relation of the isotopic frequency rule.

\section{RESULTS AND DISCUSSION}

\begin{tabular}{|c|c|c|c|}
\hline & \multirow[t]{2}{*}{$\left(\nu / \nu^{i}\right)_{\mathrm{calc}}$} & \multicolumn{2}{|c|}{$\left(\nu / \nu^{i}\right)_{\mathrm{obs}}$} \\
\hline & & Fundamentals & $\begin{array}{l}\text { Zero-order } \\
\text { frequencies }\end{array}$ \\
\hline $\begin{array}{l}\mathrm{H}_{2} \mathrm{O}, \mathrm{D}_{2} \mathrm{O}: \\
\nu_{1}\left(\nu_{*}\right)^{\mathrm{a}} \\
\nu_{2}(\delta) \\
\nu_{3}\left(\nu_{a}\right)\end{array}$ & $\begin{array}{l}1.384 \\
1.365 \\
1.365\end{array}$ & $\begin{array}{l}1.369(-1.1 \%) \\
1.348(-1.2 \%) \\
1.353(-0.9 \%)\end{array}$ & $\begin{array}{l}1.387(+0.2 \%) \\
1.363(-0.2 \%) \\
1.368(+0.2 \%)\end{array}$ \\
\hline $\begin{array}{l}\mathrm{NH}_{3}, \mathrm{ND}_{3}: \\
\nu_{1}\left(\nu_{s}\right) \\
\nu_{2}\left(\delta_{s}\right) \\
\nu_{3}\left(\nu_{a}\right) \\
\nu_{4}\left(\delta_{a}\right)\end{array}$ & $\begin{array}{l}1.395 \\
1.317 \\
1.359 \\
1.403\end{array}$ & $\begin{array}{l}1.379(-1.1 \%) \\
1.270(-3.6 \%) \\
1.350(-0.7 \%) \\
1.367(-2.6 \%)\end{array}$ & $\begin{array}{ll}1.400 & (+0.4 \%) \\
1.313 & (-0.3 \%) \\
1.357 & (-0.1 \%) \\
1.380 & (-1.6 \%)\end{array}$ \\
\hline
\end{tabular}

In order to test the validity of Eq. (10), we will consider its application to $\mathrm{H}_{2} \mathrm{O}$ and $\mathrm{NH}_{3}$. It becomes immediately apparent that in order to make use of this rule it is necessary to know the normal coordinates; only in this way can we know the relative amplitudes of motion of the atoms, and therefore the relative kinetic energies required in Eq. (10). Since such normal
TABLE I. Application of isotopic frequency rule to $\mathrm{H}_{2} \mathrm{O}$ and $\mathrm{NH}_{3}$.

a $\nu=$ stretching, $\delta=$ bending, $s=$ symmetric, $a=$ antisymmetric.

coordinates are not known in complex molecules, it would seem that the frequency rule would be of little use. However, it frequently happens that the true normal coordinate is very well approximated by a suitably chosen symmetry coordinate. For the preceding type of small molecule, the condition of conservation of momentum then uniquely defines the amplitudes of motion, and therefore permits the application of Eq. (10). The departures from the predicted frequency ratios can then serve as a measure of the departure of the true mode in the complex molecule from that represented by the simple symmetry coordinate. For $\mathrm{H}_{2} \mathrm{O}$ and $\mathrm{NH}_{3}$, symmetry coordinates were chosen in which the atoms move along and perpendicular to the bonds in the molecule, viz., valence coordinates. [See Figs. 55(b) and 58 of footnote 1 for descriptions of these symmetry coordinates.] The results of applying Eq. (10) are shown in Table I, and are compared with the ratios for the observed fundamentals and the zero-order frequencies ${ }^{6}$ (in the latter case the observed frequencies are corrected for anharmonicities). For example, for $\nu_{1}$ of $\mathrm{H}_{2} \mathrm{O}$ the relative amplitudes of motion of the $\mathrm{H}$ and $\mathrm{O}$ atoms (and therefore the $\dot{q}$ ) are in the ratio of 1 to $\frac{1}{8} \cos 52.5^{\circ}$, respectively. Therefore,

$$
\lambda_{k}{ }^{i} / \lambda_{k}=1-2 /\left[2\left(2+\frac{1}{4} \cos ^{2} 52.5\right)\right]=0.5222,
$$

and $\nu_{1} / \nu_{1}{ }^{i}=1.384$.

Several comments can be made on the results shown in Table I. First, as would be expected, the agreement is better for the zero-order frequencies than for the observed fundamentals. Second, in the cases of the $\nu_{1}, \nu_{2}$, and $\nu_{3}$ modes of $\mathrm{H}_{2} \mathrm{O}$ and the $\nu_{1}, \nu_{2}$, and $\nu_{3}$ modes of $\mathrm{NH}_{3}$ the chosen symmetry coordinate actually approximates closely the true normal coordinate, and it can be seen that the observed ratios are in quite good agreement with the calculated ones. For $\nu_{4}$ of $\mathrm{NH}_{3}$, the symmetry coordinate is, in fact, not a good approximation to the normal coordinate, and the observed ratio does deviate more significantly from the com-

${ }^{6}$ D. M. Dennison, Revs. Modern Phys. 12, 175 (1940). 
TABLE II. Frequency shift ratios for $\mathrm{CH}_{2}$ and $\mathrm{CH}_{3}$ groups.

\begin{tabular}{|c|c|c|}
\hline & & $\left(\nu / \nu^{i}\right)_{\mathrm{calc}}$ \\
\hline $\mathrm{CH}_{2}, \mathrm{CD}_{2}$ : & $\begin{array}{l}\nu_{s}{ }^{a} \\
\nu_{a} \\
\delta \\
\gamma_{v} b \\
\gamma_{t} \\
\gamma_{r}\end{array}$ & $\begin{array}{l}1.379 \\
1.349 \\
1.349 \\
1.323 \\
1.414 \\
1.379\end{array}$ \\
\hline $\mathrm{CH}_{3}, \mathrm{CD}_{3}$ : & $\begin{array}{l}\nu_{s} \\
\nu_{a} \\
\delta_{s} \\
\delta_{a} \\
\gamma_{r}\end{array}$ & $\begin{array}{l}1.398 \\
1.349 \\
1.300 \\
1.403 \\
1.292\end{array}$ \\
\hline
\end{tabular}

- See footnote to Table I.

b $\gamma_{w}=$ wagging, $\gamma_{l}=$ twisting, $\gamma_{r}=$ rocking.

puted one. However, if the true normal coordinate for $\nu_{4}$ is used in the calculation, the computed value of $\nu / \nu^{i}$ is in much better agreement with the observed value. Third, even though the ratios for the fundamental frequencies deviate somewhat more from the calculated values than do those for the zero-order frequencies, the relative values are in the same order as predicted. From the foregoing observations it can be concluded that the isotopic frequency rule given by Eq. (10) is valid to a good approximation for such small groups, and that it should provide a satisfactory basis for identifying individual modes.

Similar calculations for $\mathrm{CH}_{2}$ and $\mathrm{CH}_{3}$ groups are shown in Table II. These are again based on symmetry coordinates of the kind used for $\mathrm{H}_{2} \mathrm{O}$ and $\mathrm{NH}_{3}$. In the $\gamma_{w}, \gamma_{t}$, and $\gamma_{r}$ (wagging, twisting, and rocking) modes of $\mathrm{CH}_{2}$, motions perpendicular to the bonds have been assumed, the amplitudes being determined by the conservation of momentum condition. That is, the calculation is based on the assumption of isolated groups. In neither case can satisfactory tests of the validity of these ratios be made from the examination of small molecules, since the normal modes in such cases depart significantly from those assumed. Nevertheless, the magnitudes observed are in the order predicted. (For example, the ratio of zero-order $\mathrm{CH}_{3}$ frequencies for $\mathrm{CH}_{3} \mathrm{Cl}, \mathrm{CD}_{3} \mathrm{Cl}$ is ${ }^{7}: \nu_{s}-1.388, \nu_{a}-1.350$, $\delta_{s}-1.325, \delta_{a}-1.391, \gamma_{r}-1.320$.)

The application of the isotopic frequency rule to the spectra of complex molecules helps to establish its validity and range of application. We will consider the use of this rule in connection with the assignments of the $\nu_{s}\left(\mathrm{CH}_{2}\right), \nu_{a}\left(\mathrm{CH}_{2}\right), \delta\left(\mathrm{CH}_{2}\right), \gamma_{w}\left(\mathrm{CH}_{2}\right)$, and $\gamma_{r}\left(\mathrm{CH}_{2}\right)$ modes of some high polymers for which satisfactory data are available (the $\gamma_{t}\left(\mathrm{CH}_{2}\right)$ mode is usually hard to identify). The results for these polymers are collected in Table III. For the detailed arguments on the assignments, reference is made to a recent review article. ${ }^{8}$

\footnotetext{
${ }^{7}$ W. T. King, I. M. Mills, and B. Crawford, J. Chem. Phys. 27,455 (1957).

${ }^{8}$ S. Krimm, (to be published).
}

As we noted in the foregoing the calculated frequency ratios are for isolated $\mathrm{CH}_{2}$ groups. When a $\mathrm{CH}_{2}$ group is part of a larger molecule, it will generally be be true that atoms in the rest of the molecule also move during modes which we usually associate with only the $\mathrm{CH}_{2}$ group. To the extent that other atoms partake in the vibrational motion, we may expect the observed frequency ratios to deviate from the calculated ones given in Table III. This deviation should provide a measure of the separability of the group frequency involved. From considerations of the motions of the atoms in the infrared active modes of the polyethylene chain, we expect the relative amplitudes of motion of the atoms in the $\mathrm{CH}_{2}$ groups to approximate most closely those of an isolated $\mathrm{CH}_{2}$ group. It is therefore gratifying to find that the ratios for the $\mathrm{CH}_{2}$ modes in polyethylene most closely agree with the predicted values. (We expect some deviation because fundamental frequencies, rather than zero-order frequencies, are being used.) The larger ratio for $\gamma_{r}$ as compared to $\nu_{s}$ may indicate that in the former mode there is less carbon motion than in the latter mode, or it may be a result of different anharmonicities for the two vibrations. In polyvinyl chloride, on the other hand, the ratios as well as their relative magnitudes differ significantly from the predicted values. This may be taken to indicate that what we call a $\mathrm{CH}_{2}$ mode in this molecule actually significantly involves motions of the other atoms. That is, this is a poor instance of a separable group frequency. This situation is confirmed by other data on polyvinyl chloride: upon full deuteration of the molecule, the $\mathrm{C}-\mathrm{Cl}$ stretching frequencies shift from a mean value of $623 \mathrm{~cm}^{-1}$ to one of $581 \mathrm{~cm}^{-1}$. This $42-\mathrm{cm}^{-1}$ drop in the chlorine stretching frequencies when the hydrogen atoms are replaced by deuterium is a clear indication that the vibrational modes of this molecule significantly involve motions of all of the atoms. It would appear also that the more nearly isolated the group vibration is from motions in the remainder of the molecule the more nearly equal will be the ratios for $\nu_{a}$ and $\delta$, for example, even though the actual values deviate from the predicted ratios. Polystyrene seems to offer an illustration of this situation.

The spectrum of polyvinylidene chloride provides an illustration of how the isotopic frequency rule can be used as an aid in making assignments. Assignments for the $\nu_{s}\left(\mathrm{CH}_{2}\right), \nu_{a}\left(\mathrm{CH}_{2}\right), \delta\left(\mathrm{CH}_{2}\right)$, and $\gamma_{w}\left(\mathrm{CH}_{2}\right)$ modes in polyvinylidene chloride can be made with little diffculty, both in the normal and in the deuterated molecules. There has been some question, however, about the $\gamma_{r}\left(\mathrm{CH}_{2}\right)$ mode. One assignment would result in a frequency shift ratio of 1.240 on deuteration; an alternative assignment would give a ratio of 1.388 . Upon examining the ratios for the other $\mathrm{CH}_{2}$ modes, it is clear that the latter value is entirely reasonable, while the former is considerably out of line. Other arguments also favor the latter assignment, thus indicating that consistency with the results of the fre- 
A P POXIMATE ISOTOPIC FREQUENCY RULE

TABLE III. Application of isotopic frequency rule to some high polymers.

\begin{tabular}{|c|c|c|c|c|c|}
\hline & $v_{8}\left(\mathrm{CH}_{2}\right)$ & $\nu_{a}\left(\mathrm{CH}_{2}\right)$ & $\delta\left(\mathrm{CH}_{2}\right)$ & $\gamma_{w 0}\left(\mathrm{CH}_{2}\right)$ & $\gamma_{r}\left(\mathrm{CH}_{2}\right)$ \\
\hline Predicted ratio & 1.379 & 1.349 & 1.349 & 1.323 & 1.379 \\
\hline $\begin{array}{l}\text { Observed ratio } \\
\text { Polyethylene } \\
\text { Polyvinyl chloride } \\
\text { Polystyrene } \\
\text { Polyvinylidene chloride } \\
\text { Polyethylene terephthalate }\{\mathrm{C} \text { : } \\
\text { A: }\end{array}$ & $\begin{array}{l}1.372 \\
1.320 \\
1.358 \\
1.378 \\
1.268 \\
1.365\end{array}$ & $\begin{array}{l}1.342 \\
1.303 \\
1.330 \\
1.340 \\
1.222 \\
1.356\end{array}$ & $\begin{array}{l}1.341 \\
1.283 \\
1.334 \\
1.340 \\
1.210 \\
1.352\end{array}$ & $\begin{array}{l}\cdots \\
\cdots \\
1.322 \\
1.198(?) \\
1.302(?)\end{array}$ & $\begin{array}{l}1.384 \\
1.220 \\
\dddot{38} \\
1.271 \\
\ldots\end{array}$ \\
\hline
\end{tabular}

quency rule provides another criterion for judging whether or not an assignment is satisfactory. The results for polyethylene terephthalate are in agreement with observations made above, and indicate the possible difference between the vibrational modes of a group in the crystalline chain structure and in the amorphous chain structure.

In summary, we have been able to show that an isotopic frequency rule can be derived which is valid to a good approximation for single modes of groups within a complex molecule. Not only does the application of this rule provide information on the separability of group vibrations, but it also can be of assistence in making assignments.

\section{ACKNOWLEDGMENT}

The author is indebted to $\mathrm{K}$. T. Hecht for many helpful discussions.

\section{APPENDIX}

The relation expressed by Eq. (4), while not generally true, is valid to a good approximation for some small molecules. For diatomic molecules this is obviously the case, since in this instance

$$
\lambda=k / \mu=\left(k / m_{1}\right)+\left(k / m_{2}\right) .
$$

For a nonlinear symmetric triatomic molecule, $\mathrm{XY}_{2}$, the frequencies, on the assumption of a valence force field, are given by (see work cited in footnote 1, p. 168)

$$
\lambda_{3}=\left[1+\left(2 m_{\mathrm{Y}} / m_{\mathrm{X}}\right) \sin ^{2} \alpha\right]\left(k_{1} / m_{\mathrm{Y}}\right)
$$

$$
\begin{aligned}
& \lambda_{1}+\lambda_{2}= {\left[1+\left(2 m_{\mathrm{Y}} / m_{\mathrm{X}}\right) \cos ^{2} \alpha\right]\left(k_{1} / m_{\mathrm{Y}}\right) } \\
&+\left(2 / m_{\mathrm{Y}}\right)\left[1+\left(2 m_{\mathrm{Y}} / m_{\mathrm{X}}\right) \sin ^{2} \alpha\right]\left(k_{\delta} / l^{2}\right) \\
& \lambda_{1} \lambda_{2}=2\left[1+\left(2 m_{\mathrm{Y}} / m_{\mathrm{X}}\right)\right]\left(k_{1} / m_{\mathrm{Y}}\right) \cdot\left(k_{\delta} / l^{2}\right) .
\end{aligned}
$$

For the antisymmetric stretching frequency, given by Eq. (A2), it can be seen that a relation of the form of Eq. (4) is satisfied. From Eqs. (A3) and (A4) we find that

$\lambda_{\mathrm{I}}=\left(A / m_{\mathrm{X}}\right)+\left(B / m_{\mathrm{Y}}\right) \pm\left[\left(B^{2}-4 D\right) \cdot\left(1 / m_{\mathrm{Y}}{ }^{2}\right)\right.$

$\left.+(2 A B-4 C) \cdot\left(1 / m_{\mathrm{Y}}{ }^{2}\right) \cdot\left(m_{\mathrm{Y}} / m_{\mathrm{X}}\right)+\left(A^{2} / m_{\mathrm{X}}{ }^{2}\right)\right]^{\prime 2}$, (A5)

where $A, B, C$, and $D$ are constants involving the force constants, $k_{1}$ and $k_{\delta} / l^{2}$, and $A^{2}, B^{2}, A B, C$, and $D$ are of the same order of magnitude. Since for example for $\mathrm{CH}_{2} m_{\mathrm{X}}=12 m_{\mathrm{Y}}$, and $\left(B^{2}-4 D\right) \sim A^{2}$, the last term in the brackets of $\mathbf{E q}$. (A5) can be neglected; the error introduced is about $0.7 \%$. Expanding the bracket, and dropping terms in $\left(m_{\mathrm{Y}} / m_{\mathrm{X}}\right)^{2}$ and higher powers (since they will be negligible compared to 1 ), we find that

$$
\begin{aligned}
\lambda_{1} \cong\left[\frac{A}{m_{\mathrm{X}}} \pm \frac{\left(B^{2}-4 D\right)^{-\frac{1}{2}}(2 A B-4 C)}{m_{\mathrm{X}}}\right] \\
+\left[B \pm\left(B^{2}-4 D\right)^{\frac{1}{2}}\right] \cdot m_{\mathrm{Y}}{ }^{-1} .
\end{aligned}
$$

Since $m_{\mathrm{X}}$ is kept constant upon isotopic substitution, this can be written as

$$
\lambda_{1} \cong a_{1}+\left(a_{2} / m\right)
$$

which is of the form of Eq. (4). An analogous relationship holds for $\lambda_{2}$, and can also be shown to be similarly valid for the frequencies of a pyramidal $\mathrm{XY}_{3}$ molecule. 